\title{
Stabilization of a nonlinear system that arises in the context of vision based landing of an airliner
}

\author{
Frédéric Mazenc Laurent Burlion Victor Gibert
}

\begin{abstract}
The problem of stabilizing a nonlinear system when the variables are not accurately measured and cannot be differentiated arises when it comes to use direction measurements to one point in the environment. We here propose to adapt a recent backstepping technique with delay to the specificity of this problem. The proposed method was first motivated and thus finally applied to the vision based control problem of a landing airliner.
\end{abstract}

Index Terms - stabilization, delay, backstepping, vision-based control, aircraft.

\section{INTRODUCTION}

Recently, a significantly different backstepping design has been proposed in the papers [4] and [5]. It relies on the introduction in the expression of the control laws of artificial pointwise delays which circumvent the problem of determining Lie derivatives of the fictitious control laws used in the classical approach. Thus, it makes it possible to relax the smoothness requirement which is imposed on the fictitious control in all the previous contributions on backstepping. Moreover, for many systems of feedback or feedforward form, it can be adapted to the problem of determining stabilizing bounded feedbacks and leads to analytic expressions that are simpler than those of the feedbacks constructed in [1], [3] and [2].

The advantages of the approach of [4] led us in the present work to adapt it to a specific control design problem motivated by an engineering application on which the ANR funded VISIOLAND ${ }^{1}$ project was recently focused. Indeed, we are here interested to the automatic landing of an aircraft: the runway being unequipped, the aircraft must rely an embedded video camera which, from the control point of view, poses the problem of controlling a perspective dynamical system [12].

With the exception of [7], [8], studies which have appeared in recent years are restricted to linearized dynamics [9], [10]. Such linear systems are computed by

Mazenc is with Inria EPI DISCO, L2S-CNRS-CentraleSupélec, 3 rue Joliot Curie, 91192, Gif-sur-Yvette, France. frederic.mazenc (at) 12s.centralesupelec.fr

Burlion is with the Department of Information Processing and Systems, ONERA - The French Aerospace Lab, Toulouse, France. Burlion was partly supported by ANR VISIOLAND project ANR13-CORD-0012 dedicated to VISIOn based aircraft LANDing techniques. laurent.burlion (at) onera.fr

Gibert is with Airbus Operations, S.A.S, Toulouse, France. victor.gibert (at) airbus.com

${ }^{1}$ http://w3.onera.fr/visioland/node/92 using the runway dimensions knowledge or, in a equivalent manner, the relative altitude measurement. More recently, [11] did not assume the runway to be known but still considered a linearized longitudinal dynamics with a saturated control law. The work to follow focuses on a nonlinear class of systems which takes into account the coupling effects between longitudinal and lateral dynamics. We achieve global asymptotic stability of the system by performing a non-trivial adaptation of the designs of [4] and [5] for a model we describe in details. The numerical results show the efficiency of the control laws.

The paper is organized as follows. The main result is in Section II. It is illustrated in the specific context of vision based landing of a civil aircraft in Section III. Concluding remarks in Section IV end the paper. Technical lemmas are given in appendix.

Notation. The notation will be simplified whenever no confusion can arise from the context. Given any constant $T>0$, we let $C_{\text {in }}$ denote the set of all continuous functions $\phi:[-T, 0] \rightarrow \mathbb{R}^{a}$, which we call the set of all initial functions. We define $\Xi_{t} \in C_{\text {in }}$ by $\Xi_{t}(s)=\Xi(t+s)$ for all choices of $\Xi, s \leq 0$, and $t \geq 0$ for which the equality is defined. Given $L>0$, sat $t_{L}$ denotes the classical symmetric saturation function i.e.

$$
\operatorname{sat}_{L}(x)=\max \{-L, \min \{L, x\}\}
$$

for all $x \in \mathbb{R}$.

\section{MAin RESUlt}

\section{A. The system}

We consider the system

$$
\left\{\begin{aligned}
i_{1} & =V \cos (\gamma)\left(\tan (\gamma)-\cos (\psi) \tan \left(\gamma^{c}\right)\right) \\
i_{2} & =V \cos (\gamma) \sin (\psi) \\
\dot{\gamma} & =u_{1} \\
\dot{\psi} & =\frac{g}{V} \tan (\phi) \\
\dot{\phi} & =u_{2}
\end{aligned}\right.
$$

where $u_{1}$ and $u_{2}$ are the (scalar) control inputs, $V>0$, the constant $\gamma^{c}$ is "small" and where $\mathbb{R}^{3} \times\left(-\frac{\pi}{2}, \frac{\pi}{2}\right) \times \mathbb{R}$ is the state space. Due to the use of vision, the available outputs are:

$$
\left\{\begin{array}{l}
y_{1}(t)=\frac{1}{V} \eta(t) \iota_{1}(t) \\
y_{2}(t)=\frac{1}{V} \eta(t) \iota_{2}(t) \\
y_{3}(t)=\gamma(t) \\
y_{4}(t)=\psi(t) \\
y_{5}(t)=\phi(t)
\end{array}\right.
$$


where $\eta$ is not known, piecewise continuous and $\eta(t) \in$ $[\underline{\eta}, \bar{\eta}]$ for all $t \geq 0, \underline{\eta}>0, \bar{\eta}>\underline{\eta}$ being two known constants.

The change of coordinates $x_{1}=\frac{\cos \left(\gamma^{c}\right)}{V} \iota_{1}(t)$ and $x_{2}=$ $\frac{1}{V} \iota_{2}(t)$ and the fact that

$$
\dot{x}_{1}=\sin \left(\gamma-\gamma^{c}\right)+\cos (\gamma) \sin \left(\gamma^{c}\right)(1-\cos (\psi))
$$

give

$$
\left\{\begin{aligned}
\dot{x}_{1} & =\sin \left(x_{3}\right)+\cos \left(x_{3}+\gamma^{c}\right) \sin \left(\gamma^{c}\right)[1-\cos (\psi)] \\
\dot{x}_{3} & =u_{1} \\
\dot{x}_{2} & =\cos \left(x_{3}+\gamma^{c}\right) \sin (\psi) \\
\dot{\psi} & =\frac{g}{V} \tan (\phi) \\
\dot{\phi} & =u_{2}
\end{aligned}\right.
$$

with $x_{3}=\gamma-\gamma^{c}$. System (5) has a specific structure, which implies that when the function $\eta(t)$ is known and differentiable, then the system (5) can be stabilized by feedbacks that provides the classical backstepping approach. This technique requires first the stabilization of

$$
\left\{\begin{array}{l}
\dot{x}_{1}=\sin \left(\nu_{1}\right)+\cos \left(\nu_{1}+\gamma^{c}\right) \sin \left(\gamma^{c}\right)\left[1-\cos \left(\nu_{2}\right)\right] \\
\dot{x}_{2}=\cos \left(\nu_{1}+\gamma^{c}\right) \sin \left(\nu_{2}\right)
\end{array}\right.
$$

with $\nu_{1}$ and $\nu_{2}$ as input and next consists in the addition of integrators [6]. But when $\eta(t)$ is not known and possibly not differentiable, then, to the best of our knowledge, no result available in the literature enables to construct stabilizing control laws.

\section{B. Global asymptotic stabilization}

1) Preliminary step: Let us perform a change of feedback:

$$
u_{1}=\frac{-r_{1} \sin \left(\sigma_{1}\left(x_{3}\right)\right)+v_{1}}{\cos \left(\sigma_{1}\left(x_{3}\right)\right)}
$$

where $r_{1}>0$,

$$
\sigma_{1}=\operatorname{sat}_{\frac{\pi}{3}} \quad \text { and } \quad\left|v_{1}\right|_{\infty}<\frac{r_{1}}{4}
$$

Now, let us apply the change of variable to (5) with (7)

$$
x_{4}=\frac{g}{V} \tan (\phi)
$$

that is defined for all $\phi \in\left(-\frac{\pi}{2}, \frac{\pi}{2}\right)$. It gives

$$
\left\{\begin{aligned}
\dot{x}_{1} & =\sin \left(x_{3}\right)+\cos \left(x_{3}+\gamma^{c}\right) \sin \left(\gamma^{c}\right)(1-\cos (\psi)) \\
\dot{x}_{3} & =\frac{-r_{1} \sin \left(\sigma_{1}\left(x_{3}\right)\right)+v_{1}}{\cos \left(\sigma_{1}\left(x_{3}\right)\right)} \\
\dot{x}_{2} & =\cos \left(x_{3}+\gamma^{c}\right) \sin \psi \\
\dot{\psi} & =x_{4} \\
\dot{x}_{4} & =\frac{g}{V}\left(1+\frac{V^{2}}{g^{2}} x_{4}^{2}\right) u_{2}
\end{aligned}\right.
$$

whose state space is $\mathbb{R}^{5}$.

Let us point out that if we can construct a globally asymptotically stabilizing feedback for the origin of (10), then we will obtain a feedback that ensures that $\mathbb{R}^{3} \times$ $\left(-\frac{\pi}{2}, \frac{\pi}{2}\right) \times \mathbb{R}$ is the basin of attraction of the origin of (5).
Now, the change of feedback

$$
u_{2}=\frac{V}{g} \frac{u_{3}}{1+\frac{V^{2}}{g^{2}} x_{4}^{2}}
$$

gives

$$
\left\{\begin{aligned}
\dot{x}_{1} & =\sin \left(x_{3}\right)+\cos \left(x_{3}+\gamma^{c}\right) \sin \left(\gamma^{c}\right)(1-\cos (\psi)) \\
\dot{x}_{3} & =\frac{-r_{1} \sin \left(\sigma_{1}\left(x_{3}\right)\right)+v_{1}}{\cos \left(\sigma_{1}\left(x_{3}\right)\right)} \\
\dot{x}_{2} & =\cos \left(x_{3}+\gamma^{c}\right) \sin (\psi) \\
\dot{\psi} & =x_{4} \\
\dot{x}_{4} & =u_{3}
\end{aligned}\right.
$$

Notice that this system is forward complete because the nonlinear terms of the system are bounded.

Now, let us establish that any solution of (12) is such that $x_{3}(t)$ enters the interval $\left[-\arcsin \left(\frac{1}{4}\right), \arcsin \left(\frac{1}{4}\right)\right]$ in finite time. When $x_{3}(t) \geq \arcsin \left(\frac{1}{4}\right)$, then, from (8), we deduce that

$$
\dot{x}_{3}(t) \leq \frac{-r_{1} \sin \left(\sigma_{1}\left(\arcsin \left(\frac{1}{4}\right)\right)+v_{1}\right.}{\cos \left(\sigma_{1}\left(x_{3}(t)\right)\right)} \leq-\frac{r_{1}}{4}+\left|v_{1}\right|_{\infty}<0
$$

Similarly, one can show that when $x_{3}(t) \leq-\arcsin \left(\frac{1}{4}\right)$, then $\dot{x}_{3}(t) \geq \frac{r_{1}}{4}-\left|v_{1}\right|_{\infty}>0$. We deduce that there is a constant $t_{a} \geq 0$ such that for all $t \geq t_{a}, x_{3}(t) \in$ $\left[-\arcsin \left(\frac{1}{4}\right), \arcsin \left(\frac{1}{4}\right)\right]$. It straightforwardly follows that, for all $t \geq t_{a},\left|x_{3}(t)+\gamma^{c}\right| \leq \arcsin \left(\frac{1}{4}\right)+\gamma^{c}$. Then

$$
\cos \left(x_{3}(t)+\gamma^{c}\right) \geq \cos \left(\arcsin \left(\frac{1}{4}\right)+\gamma^{c}\right) \geq \frac{1}{2}
$$

for all $t \geq t_{a}$. This fact is fundamental: it implies that if we can globally asymptotically stabilize the origin of the system

$$
\left\{\begin{aligned}
\dot{x}_{2} & =\varpi(t) \sin (\psi) \\
\dot{\psi} & =x_{4} \\
\dot{x}_{4} & =u_{3}
\end{aligned}\right.
$$

for any continuous function such that $\varpi(t) \in\left[\frac{1}{2}, 1\right]$ for all $t \geq 0$ and globally asymptotically stabilize the origin of the system

$$
\left\{\begin{array}{l}
\dot{x}_{1}=\sin \left(x_{3}\right)+d_{1}(t) \\
\dot{x}_{3}=\frac{-r_{1} \sin \left(\sigma_{1}\left(x_{3}\right)\right)+v_{1}}{\cos \left(\sigma_{1}\left(x_{3}\right)\right)}
\end{array}\right.
$$

where $d_{1}$ is a continuous function such that $\lim _{t \rightarrow+\infty}\left|d_{1}(t)\right|=0$, then we can globally asymptotically stabilize the origin of the system (2) with $\mathbb{R}^{3} \times\left(-\frac{\pi}{2}, \frac{\pi}{2}\right) \times \mathbb{R}$ as state space.

2) Stabilization of the system (16): From Section IIB.1, we deduce that there is a constant $t_{b} \geq 0$ such that for all $t \geq t_{b},\left|x_{3}(t)\right| \leq \arcsin \left(\frac{1}{4}\right)$ and then

$$
\left\{\begin{array}{l}
\dot{x}_{1}=\sin \left(x_{3}\right)+d_{1}(t) \\
\dot{x}_{3}=\frac{-r_{1} \sin \left(x_{3}\right)+v_{1}}{\cos \left(x_{3}\right)}
\end{array}\right.
$$

Let $z_{1}=\sin \left(x_{3}\right)$. Then

$$
\left\{\begin{array}{c}
\dot{x}_{1}=z_{1}+d_{1}(t) \\
\dot{z}_{1}=-r_{1} z_{1}+v_{1}
\end{array}\right.
$$


Then, $v_{1}$ is designed following the result presented in Appendix A.

3) Stabilization of the system (15): Let $\psi=\psi_{1}$ and $\psi_{2}=x_{4}+c_{1} \psi$ where $c_{1}>0$ is to be selected later. We have

$$
\left\{\begin{aligned}
\dot{x}_{2} & =\varpi(t) \sin \left(\psi_{1}\right) \\
\dot{\psi}_{1} & =-c_{1} \psi_{1}+\psi_{2} \\
\dot{\psi}_{2} & =u_{3}-c_{1}^{2} \psi_{1}+c_{1} \psi_{2}
\end{aligned}\right.
$$

Let us perform a change of feedback:

$$
u_{3}=-\left(c_{1}+c_{2}\right) x_{4}-c_{1} c_{2} \psi_{1}+v_{3}
$$

where $c_{2}>0$ Then

$$
\left\{\begin{array}{l}
\dot{x}_{2}=\varpi(t) \sin \left(\psi_{1}\right) \\
\dot{\psi}_{1}=-c_{1} \psi_{1}+\psi_{2} \\
\dot{\psi}_{2}=-c_{2} \psi_{2}+v_{3}
\end{array}\right.
$$

Then, $v_{3}$ is designed following the result presented in Appendix B.

\section{ILluStration}

\section{A. Landing aircraft dynamics}

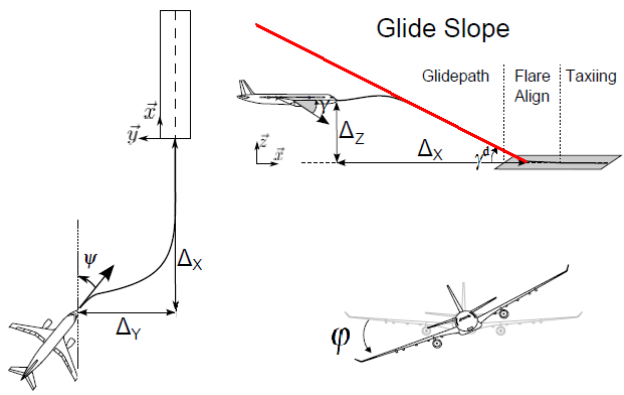

Fig. 1. Landing phases

In this work, we focus on the glide slope phase (see figure 1) during which an autopilot system must maintain a constant glide slope $\gamma^{c}=-3 d e g$ at a constant airspeed $V=70 \mathrm{~ms}^{-1}$. Let us note $\Delta_{X}, \Delta_{Y}, \Delta_{Z}$ the vector components between the aircraft center of gravity and the runway touchdown point. The velocity vector is given by:

$$
\left\{\begin{array}{l}
\dot{\Delta}_{X}=V \cos (\gamma) \cos (\psi) \\
\dot{\Delta}_{Y}=V \cos (\gamma) \sin (\psi) \\
\dot{\Delta}_{Z}=V \sin (\gamma)
\end{array}\right.
$$

where $\gamma($ resp. $\psi)$ is the aircraft relative slope (resp. yaw) with respect to the runway. In addition, we have:

$$
\left\{\begin{aligned}
\dot{\gamma} & =u_{1} \\
\dot{\psi} & =\frac{g}{V} \tan (\phi) \\
\dot{\phi} & =u_{2}
\end{aligned}\right.
$$

in which $\phi$ is the aircraft roll angle, $g=9.81 m . s^{-2}$ is the local gravity and $\left[u_{1}, u_{2}\right]^{T}$ is the guidance control input vector.
The glide slope phase consists in tracking the line defined by $\Delta_{Z}^{c}=\tan \left(\gamma^{c}\right) \Delta_{X}$, the deviations are computed as follows:

$$
\left\{\begin{aligned}
\iota_{1} & =\Delta_{Z}-\Delta_{Z}^{d} \\
\iota_{2} & =\Delta_{Y}
\end{aligned}\right.
$$

Now, observe that for $\gamma \in]-\frac{\pi}{2}, \frac{\pi}{2}[$ :

$$
\begin{aligned}
i_{1}= & V \sin (\gamma)-V \tan \left(\gamma^{c}\right) \cos (\gamma) \cos (\psi) \\
= & V \tan (\gamma) \cos (\gamma)-V \tan \left(\gamma^{c}\right) \cos (\gamma) \cos (\psi) \\
= & V \cos (\gamma)\left(\tan (\gamma)-\tan \left(\gamma^{c}\right)\right) \\
& +V \tan \left(\gamma^{c}\right) \cos (\gamma)(1-\cos (\psi))
\end{aligned}
$$

From (24)-(22)-(23)-(25), it is now clear that these deviations dynamics served to define our class of systems (2).

\section{B. Available measurements in a vision based guidance scenario}

Similarly, let us now explain how we obtain the available outputs (3).

Consider the vision based control problem on which the ANR funded VISIOLAND ${ }^{2}$ project was focused. In this project, the most stringent scenario (from the control point of view) is the vision based landing of an aircraft on an unequipped runway whose size is partially unknown. More precisely, the runway is unequipped in the sense that there are no landing ground facilities as ILS (Instrument Landing System) or GPS (Global Positioning System). As such, an automatic guidance loop must merely rely on embedded sensors which, in this case, were a monocular camera and an Inertial Measurement Unit (IMU). Assuming the runway is not inclined, the relative attitude angles $y_{3}=\gamma$ and $y_{5}=\phi$ are simply the aircraft's yaw and roll angles, thus given by the IMU.

Let $w$ denote the runway width. Assuming the runway is kept inside the camera field of view all along the descent, it was shown in [14] that the relative yaw angle $y_{4}=\psi$ and the outputs $y_{\text {image }, 1}=-\frac{\Delta_{Z}}{\Delta_{X}}, y_{\text {image }, 2}=$ $-\frac{w}{\Delta_{X}}, y_{\text {image }, 3}=-\frac{\Delta_{Y}}{\Delta_{X}}$ can be computed applying some image processing and a 'derotation' transformation to each image given by the body fixed camera.

Let us note $\hat{w}_{X}$ a rough estimate of $w$. It can be either a constant value chosen in the standard interval $[30 \mathrm{~m}, 60 \mathrm{~m}]$ or the saturated output of a width estimator [11]). Let $\eta=\frac{\hat{w}}{w}, \iota_{1}=\Delta_{Z}-\tan \left(\gamma^{d}\right) \Delta_{X}$ and $\iota_{2}=\Delta_{Y}$. It is not difficult to see that the outputs $y_{1}$ and $y_{2}$ can be computed from the definition of $y_{\text {image, } 1}$ to $y_{\text {image, } 3}$. Indeed, using (3), observe that:

$$
\begin{aligned}
y_{1} & =\frac{\eta}{V} \iota_{1}=\frac{\hat{w}}{V y_{\text {image }, 2}}\left(y_{\text {image }, 1}+\tan \left(\gamma^{d}\right)\right) \\
y_{2} & =\frac{\eta}{V} \iota_{2}=\frac{\hat{w}}{V y_{\text {image }, 2}} y_{\text {image }, 3}
\end{aligned}
$$

${ }^{2}$ http://w3.onera.fr/visioland/node/92 
Now, let us detail the control laws given the following parameters: $V=70 \mathrm{~m} . \mathrm{s}^{-1} ; g=9.81 \mathrm{~m} . \mathrm{s}^{-2} ; \bar{\eta}=1.5 ; \underline{\eta}=0.5$

\section{Longitudinal control law tuning}

Let us here recall the obtained control law (7)-(34):

$$
u_{1}(t)=\frac{-r_{1}\left(\sin \left(\sigma_{3}\left(y_{1}(t)\right)\right)+\sigma_{3}\left(y_{1}\right)\right)}{\cos \left(\sigma_{3}\left(y_{1}(t)\right)\right)}
$$

with $\sigma_{3}=l_{1} s_{l_{2}}$. Also, recall that in virtue of equations (35), (36), (37), the positive constants $r_{1}, l_{1}, l_{2}$ must satisfy

$$
l_{1} l_{2} \leq \frac{1}{4} \quad, \quad r_{1} \geq 2 \bar{\eta} \quad, \quad l_{1} \leq \frac{9}{20 \bar{\eta}}
$$

We thus propose to use the following set of parameters which satisfy such inequalities: $r_{1}=3, l_{1}=0.3$ and $l_{2}=$ 0.83 .

\section{Lateral control law tuning}

Let us here recall the obtained dynamical law (11)(20)-(63)-(50)-(57)-(58):

$$
\begin{gathered}
u_{2}=\frac{\frac{V}{g}}{1+\frac{V^{2}}{g^{2}} x_{4}^{2}}\left[-\left(c_{1}+c_{2}\right) x_{4}-c_{1} c_{2} \psi_{1}+c_{2} c_{1} \mathcal{F}\left(z_{1, t}\right)\right. \\
\left.+\left(c_{1}+c_{2}\right) \mathcal{G}\left(z_{1, t}, z_{2, t}\right)+\mathcal{H}\left(t, z_{1, t}, z_{2, t}, x_{2, t}\right)\right](28) \\
\left\{\begin{aligned}
\dot{\mathcal{F}}\left(z_{1, t}\right) & =\mathcal{G}\left(z_{1, t}, z_{2, t}\right) \\
\dot{\mathcal{G}}\left(z_{1, t}, z_{2, t}\right) & =\mathcal{H}\left(t, z_{1, t}, z_{2, t}, x_{2, t}\right) \\
\dot{z}_{1}(t) & =q\left[-z_{1}(t)+z_{2}(t)\right] \\
\dot{z}_{2}(t) & =q\left[-z_{2}(t)-\sigma_{\dagger}\left(y_{2}(t)\right)\right]
\end{aligned}\right.
\end{gathered}
$$

with $c_{1}, c_{2}>0, x_{4}=\frac{g}{V} \phi, \psi_{1}=\psi, \sigma_{\dagger}=\varsigma_{1} s_{a} t_{\varsigma_{2}} \varsigma_{3}$ and $\mathcal{H}$ given by (55).

Let us recall that the positive constants $\tau, \varsigma_{1}, \varsigma_{2}, \varsigma_{3}$ must satisfy

$$
\begin{gathered}
\tau^{6}<\frac{\underline{\underline{\theta}} \underline{\left(1-e^{-q \tau}\right)^{4}}}{16 \bar{\theta}^{3} \varsigma_{1}^{2} \varsigma_{3}^{2} \bar{\eta}^{3} q^{4}} \\
\varsigma_{1} \varsigma_{2} \leq \frac{\pi}{4}
\end{gathered}
$$

We then propose the following empirical procedure to choose the control law parameters.

1) First consider the state feedback case (i.e the case where $\eta=\bar{\eta})$ ). Then, by letting $\tau$ go to 0 , we approximate $\mathcal{F}\left(z_{1, t}\right)$ by the linear term $-\varsigma_{1} \varsigma_{3} x_{2}$. Thus, in the state feedback case, the linearized system is:

$$
\begin{aligned}
\dot{x}_{2}= & \psi \\
\dot{\psi}= & x_{4} \\
\dot{x}_{4}= & -\left(c_{1}+c_{2}\right)\left(x_{4}+\varsigma_{1} \varsigma_{3} \psi\right)-c_{1} c_{2}\left(\psi_{1}+\varsigma_{1} \varsigma_{3} x_{2}\right) \\
& -\varsigma_{1} \varsigma_{3} x_{4}
\end{aligned}
$$

Then, choose the positive constants $c_{1}, c_{2}, \varsigma_{1}, \varsigma_{3}$ to fulfill some requirements on the linearized closed loop dynamics.
2) $\varsigma_{1}$ being chosen, compute $\varsigma_{2}=\frac{\pi}{4 \varsigma_{1}}$ and choose $q$ and $\tau$ to satisfy $(30)$

First, using $\underline{\theta}=\frac{2 \sqrt{2}}{\pi}$ and $\bar{\theta}=1$, one has:

$$
\tau^{6}<\frac{\frac{\sqrt{2}}{\pi}\left(1-e^{-q \tau}\right)^{4}}{128 \varsigma_{1}^{2} \varsigma_{3}^{2} q^{4}}
$$

and then $q=0.5$ yields:

$$
\tau^{6}<1.27\left(1-e^{-0.5 \tau}\right)^{4}
$$

which holds when $\tau \leq 0.25$

As a result, our tuning process ended up with the following parameters: $c_{1}=0.2, c_{2}=0.6, \varsigma_{1}=0.003, \varsigma_{2}=78.5$, $\varsigma_{3}=70, q=0.5, \tau=0.25$.

\section{E. Numerical results}

Numerical simulations are performed starting from $\psi=45 \mathrm{deg}$ (which is sufficiently large so that the nonlinear coupling terms cannot be neglected). We consider several constant values for $\eta$. We also consider the case where $\eta$ is time-varying e.g. $\eta(t)=1-0.5 e^{-0.1 t}$. The results are presented in Figures 2 and 3 and show that the proposed control laws are able to stabilize the aircraft on its glide slope for all the studied $\eta$ values.

Note that we increased the value of $\tau$ from 0.25 to 3 in order to obtain a less aggressive lateral control law. Unsurprisingly, this reveals that the established bound for the maximum value of $\tau$ is conservative and we thus suggest to increase its value in practice.

\section{CONCLusion}

In this paper, recent backstepping approaches [4], [5] have been tailored to solve a non-trivial visual servoing control design problem. The key ingredient of the approach was to use a finite dimensional dynamic extension to circumvent the problem posed by the use of visual measurements. Indeed, classical Backstepping requires the successive pseudo-controllers to be sufficiently smooth which was not the case in our application. In the future, we plan to extend our results to allow the use of sampled and delayed visual informations, taking thus into account the image processing in our design.

\section{REFERENCES}

[1] R. Freeman, L. Praly, Integrators backstepping for bounded controls and control rates, IEEE Trans. Automat. Control, 43 (1998), pp. 258-262.

[2] F. Mazenc, S. Bowong, Backstepping with bounded feedbacks for time-varying systems. SIAM Journal on Control and Optimization, Vol. 43, No. 3, pp. 856-871, 2004.

[3] F. Mazenc, A. Iggidr, Backstepping with Bounded Feedbacks. Systems and Control Letters, Vol. 51/3-4, pp. 235-245, 2004.

[4] F. Mazenc, M. Malisoff, New Control Design for Bounded Backstepping under Input Delay. Automatica, vol. 66, pp. 4855, April 2016.

[5] F. Mazenc, M. Malisoff, J. Weston, New bounded backstepping control designs for time-varying systems under converging input converging state conditions. in Proceedings of the 55th IEEE Conference on Decision and Control, pp. 3167-3171, Las Vegas, NV, USA, Dec. 2016.

[6] R. Sepulchre, M. Jankovic, P. V. Kokotovic, Constructive Nonlinear Control, Springer-Verlag, Berlin, 1996. 

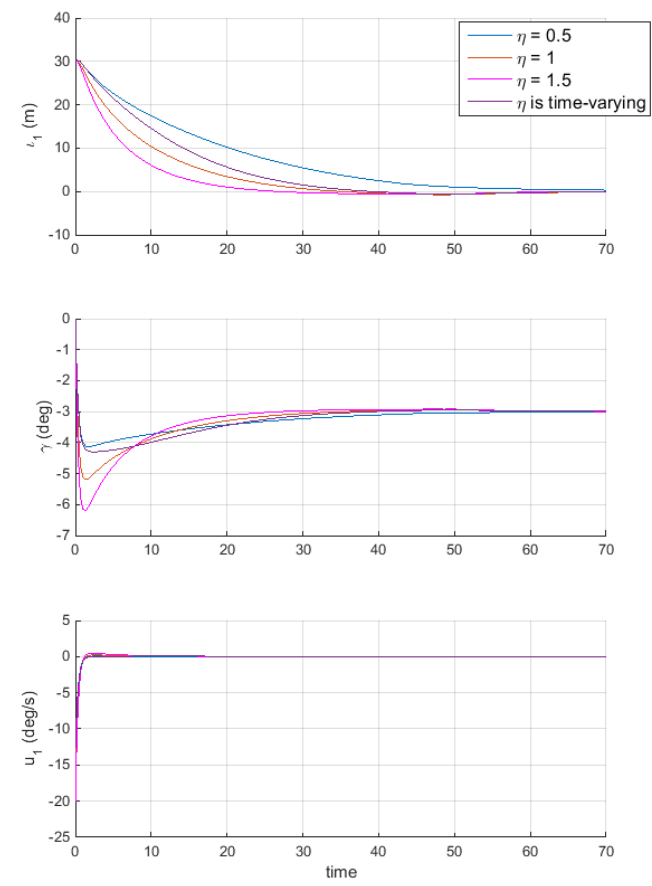

Fig. 2. Longitudinal states evolution

[7] F. Le Bras, T. Hamel, R. Mahony, C. Barat and J. Thadasack, Approach maneuvers for autonomous landing using visual servo control, IEEE Transactions on Aerospace and Electronic Systems, vol. 50, no. 2, pp. 1051-1065, April 2014.

[8] P. Serra, R. Cunha, T. Hamel, D. Cabecinhas and C. Silvestre, Landing of a Quadrotor on a Moving Target Using Dynamic Image-Based Visual Servo Control, IEEE Transactions on Robotics, vol. 32, no. 6, pp. 1524-1535, Dec. 2016.

[9] O. Bourquardez and F. Chaumette, Visual servoing of an airplane for auto-landing, 2007 IEEE/RSJ International Conference on Intelligent Robots and Systems, San Diego, CA, pp. 1314-1319, 2007.

[10] L. Coutard, F. Chaumette and J. M. Pflimlin, Automatic landing on aircraft carrier by visual servoing, 2011 IEEE/RSJ International Conference on Intelligent Robots and Systems, San Francisco, CA, pp. 2843-2848, 2011.

[11] L. Burlion, H. de Plinval, Vision based anti-windup design with application to the landing of an Airliner, 20th IFAC World Congress, pp.10482-10487, Toulouse, France, 2017.

[12] D. Karagiannis, A. Astolfi, A new solution to the problem of range identification in perspective vision systems, IEEE Trans. Automat. Contr., 50 (12) : 2074-2077, 2005.

[13] V. Gibert, L. Burlion, A. Chriette, J.Boada, F. Plestan, Nonlinear observers in vision system: Application to civil aircraft landing, European Control Conference, pp. 1818-1823, Linz, Austria, 2015.

[14] L. Burlion and H. de Plinval, Toward vision based landing of a fixed-wing UAV on an unknown runway under some fov constraints, 2017 International Conference on Unmanned Aircraft Systems (ICUAS), pp. 1824-1832, Miami, Florida, 2017.
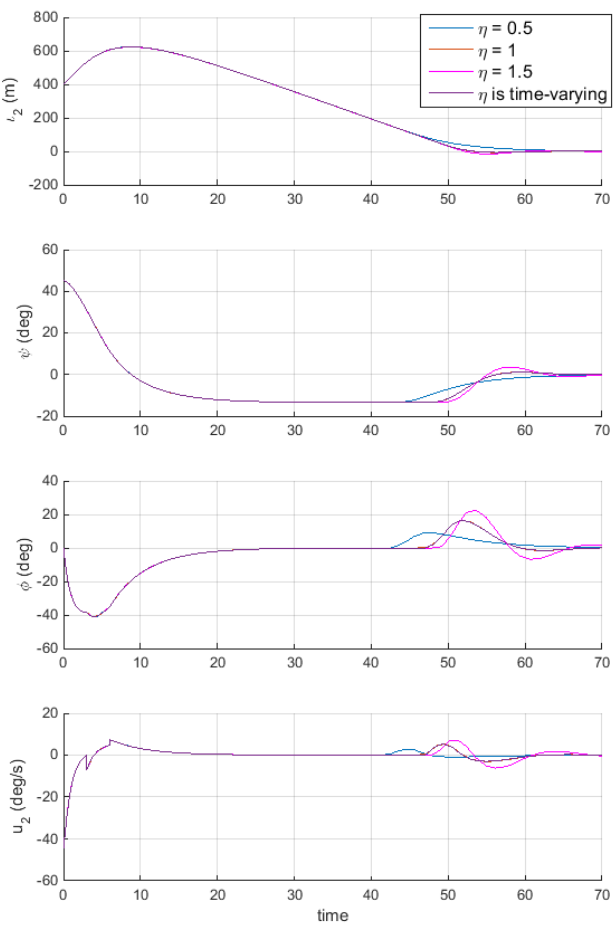

Fig. 3. Lateral states evolution (when $\tau=3 \mathrm{~s}$ )

\section{APPENDix}

\section{A. First result}

Consider the system

$$
\left\{\begin{array}{l}
\dot{x}_{1}(t)=z_{1}(t)+d_{1}(t) \\
\dot{z}_{1}(t)=-r_{1} z_{1}(t)+v_{1}(t)
\end{array}\right.
$$

where $r_{1}>0$ is a constant, $v_{1}$ is the control, $d_{1}$ is a disturbance and the measurement is $y_{1}(t)=\eta(t) x_{1}(t)$.

If $\eta(t)$ is known, then stabilizing (33) by bounded feedback is easy. But when $\eta(t)$ is unknown, more care must be taken to demonstrate a similar result. Let us choose:

$$
v_{1}=-r_{1} \sigma_{3}\left(y_{1}(t)\right)
$$

with $\sigma_{3}=l_{1} s a t_{l_{2}}, l_{1}>0, l_{2}>0$ such that

$$
l_{1} l_{2} \leq \frac{1}{4}
$$

in order to satisfy (8) and

$$
2 \bar{\eta} \leq r_{1}
$$

and

$$
\frac{20 \bar{\eta} l_{1}}{9} \leq 1
$$


Then, let us study the stability of the system:

$$
\left\{\begin{array}{l}
\dot{x}_{1}(t)=z_{1}(t)+d_{1}(t) \\
\dot{z}_{1}(t)=-r_{1}\left(z_{1}(t)+\sigma_{3}\left(\eta(t) x_{1}(t)\right)\right)
\end{array}\right.
$$

Let us introduce a new variable:

$$
m(t)=x_{1}(t)+\frac{1}{r_{1}} z_{1}(t)
$$

Then

$$
\left\{\begin{array}{l}
\dot{m}(t)=-\sigma_{3}\left(\eta(t)\left(m(t)-\frac{1}{r_{1}} z_{1}(t)\right)\right)+d_{1}(t) \\
\dot{z}_{1}(t)=r_{1}\left[-z_{1}(t)-\sigma_{3}\left(\eta(t)\left(m(t)-\frac{1}{r_{1}} z_{1}(t)\right)\right)\right]
\end{array}\right.
$$

it is easy to see that there exists $t_{c}$ such that when $t \geq t_{c}$,

$$
\left|z_{1}(t)\right| \leq l_{1} l_{2}
$$

Since $\lim _{t \rightarrow+\infty}\left|d_{1}(t)\right|=0$, one can easily prove using (40) that there is $t_{d} \geq t_{c}$ such that for all $t \geq t_{d}$ then

$$
|m(t)| \leq \frac{11}{9 r_{1}} l_{1} l_{2}
$$

This inequality and (41) give

$$
\eta(t)\left|m(t)-\frac{1}{r_{1}} z_{1}(t)\right| \leq \frac{20 \bar{\eta}}{9 r_{1}} l_{1} l_{2}
$$

for all $t \geq t_{d}$. Therefore, from (37), it follows that $\left|\eta(t)\left(m(t)-\frac{1}{r_{1}} z_{1}(t)\right)\right| \leq l_{2}$ for all $t \geq t_{d}$, which implies that

$$
\sigma_{3}\left(\eta(t)\left(m(t)-\frac{1}{r_{1}} z_{1}(t)\right)\right)=\eta(t)\left(m(t)-\frac{1}{r_{1}} z_{1}(t)\right)
$$

Then

$$
\left\{\begin{array}{l}
\dot{m}(t)=-\eta(t)\left(m(t)-\frac{1}{r_{1}} z_{1}(t)\right)+d_{1}(t) \\
\dot{z}_{1}(t)=p\left[-z_{1}(t)-\eta(t)\left(m(t)-\frac{1}{r_{1}} z_{1}(t)\right)\right]
\end{array}\right.
$$

It rewrites as

$$
\left\{\begin{array}{l}
\dot{m}(t)=-\eta(t) m(t)+\frac{\eta(t)}{r_{1}} z_{1}(t)+d_{1}(t) \\
\dot{z}_{1}(t)=\left(\eta(t)-r_{1}\right) z_{1}(t)-p \eta(t) m(t)
\end{array}\right.
$$

Now, we analyze the stability of this system via the candidate Lyapunov function:

$$
\nu\left(m, z_{1}\right)=\frac{r_{1}^{2}}{2} m^{2}+\frac{1}{2} z_{1}^{2}
$$

Elementary calculations give

$$
\begin{aligned}
\dot{\nu}(t)= & r_{1}^{2} m(t)\left[-\eta(t) m(t)+\frac{\eta(t)}{r_{1}} z_{1}(t)+d_{1}(t)\right] \\
& +z_{1}(t)\left[\left(\eta(t)-r_{1}\right) z_{1}(t)-r_{1} \eta(t) m(t)\right] \\
= & -\eta(t) r_{1}^{2} m(t)^{2}+\left(\eta(t)-r_{1}\right) z_{1}(t)^{2} \\
& +r_{1}^{2} m(t) d_{1}(t)
\end{aligned}
$$

From (36), we deduce that

$$
\dot{\nu}(t) \leq-\frac{\eta}{2} r_{1}^{2} m(t)^{2}-\frac{r_{1}}{2} z_{1}(t)^{2}+\frac{1}{2 \underline{\eta}} r_{1}^{2} d_{1}(t)^{2}
$$

It follows that $\lim _{t \rightarrow+\infty}\left|d_{1}(t)\right|=0$ ensures that all the solutions of (45) converge to the origin. This allows us to conclude that all the solutions of the system (38) go to the origin when $\lim _{t \rightarrow+\infty}\left|d_{1}(t)\right|=0$.

\section{B. Second result}

We consider the system

$$
\left\{\begin{array}{l}
\dot{x}_{2}=\varpi(t) \sin \left(\psi_{1}\right) \\
\dot{\psi}_{1}=-c_{1} \psi_{1}+\psi_{2} \\
\dot{\psi}_{2}=-c_{2} \psi_{2}+v_{3}
\end{array}\right.
$$

We introduce a dynamic extension:

$$
\left\{\begin{array}{l}
\dot{z}_{1}(t)=q\left[-z_{1}(t)+z_{2}(t)\right] \\
\dot{z}_{2}(t)=q\left[-z_{2}(t)-\sigma_{\dagger}\left(y_{2}(t)\right)\right]
\end{array}\right.
$$

where $q>0$ is a constant and

$$
\sigma_{\dagger}=\varsigma_{1} s a t_{\varsigma_{2}} \varsigma_{3}
$$

with $\varsigma_{3}>0$ and with $\varsigma_{1}>0$ and $\varsigma_{2}>0$ are chosen such that:

$$
\varsigma_{1} \varsigma_{2} \leq \frac{\pi}{4}
$$

Now, let us introduce several functionals:

$$
\mathcal{F}\left(z_{1, t}\right)=c_{\triangle}\left[z_{1}(t)-2 e^{-q \tau} z_{1}(t-\tau)+e^{-2 q \tau} z_{1}(t-2 \tau)\right]
$$

with $c_{\triangle}=\frac{1}{\left(1-e^{-q \tau}\right)^{2}}$,

$$
\begin{aligned}
\mathcal{G}\left(z_{1, t}, z_{2, t}\right)= & c \triangle q\left[-z_{1}(t)+z_{2}(t)+2 e^{-q \tau}\left(z_{1}(t-\tau)\right.\right. \\
& \left.-z_{2}(t-\tau)\right)+e^{-2 q \tau}\left(-z_{1}(t-2 \tau)\right. \\
& \left.\left.+z_{2}(t-2 \tau)\right)\right]
\end{aligned}
$$

and

$$
\begin{aligned}
& \mathcal{H}\left(t, z_{1, t}, z_{2, t}, x_{2, t}\right)=c_{\triangle} q^{2}\left[z_{1}(t)-2 z_{2}(t)\right. \\
& -2 e^{-q \tau} z_{1}(t-\tau)+e^{-2 q \tau} z_{1}(t-2 \tau)-\sigma_{\dagger}\left(y_{2}(t)\right) \\
& +2 e^{-q \tau} \sigma_{\dagger}\left(y_{2}(t-\tau)\right)-e^{-2 q \tau} \sigma_{\dagger}\left(y_{2}(t-2 \tau)\right) \\
& \left.+4 e^{-q \tau} z_{2}(t-\tau)-2 e^{-2 q \tau} z_{2}(t-2 \tau)\right]
\end{aligned}
$$

Notice for later use that, for all $t \in \mathbb{R}$,

$$
c_{\triangle}=\frac{1}{q^{2} \int_{t-\tau}^{t} e^{q(m-t)} \int_{m-\tau}^{m} e^{q(\ell-m)} d \ell d m}
$$

and

$$
\begin{aligned}
\dot{\mathcal{F}}(t)= & \mathcal{G}\left(z_{1, t}, z_{2, t}\right) \\
\dot{\mathcal{F}}(t) & -\dot{z}_{1}(t)+2 e^{-q \tau} \dot{z}_{1}(t-\tau)-e^{-2 q \tau} \dot{z}_{1}(t-2 \tau) \\
& +\dot{z}_{2}(t)-2 e^{-q \tau} \dot{z}_{2}(t-\tau)+e^{-2 q \tau} \dot{z}_{2}(t-2 \tau) \\
= & q\left[z_{1}(t)-z_{2}(t)\right]-2 e^{-q \tau} q\left[z_{1}(t-\tau)-z_{2}(t-\tau)\right] \\
& +e^{-2 q \tau} q\left[z_{1}(t-2 \tau)-z_{2}(t-2 \tau)\right] \\
& +q\left[-z_{2}(t)-\sigma_{\dagger}\left(y_{2}(t)\right)\right]+2 e^{-q \tau} q\left[z_{2}(t-\tau)\right. \\
& \left.+\sigma_{\dagger}\left(y_{2}(t-\tau)\right)\right]+e^{-2 q \tau} q\left[-z_{2}(t-2 \tau)\right. \\
& \left.-\sigma_{\dagger}\left(y_{2}(t-2 \tau)\right)\right]
\end{aligned}
$$

Thus

$$
\ddot{\mathcal{F}}(t)=\mathcal{H}\left(t, z_{1, t}, z_{2, t}, x_{2, t}\right)
$$


Now, let

$$
\psi_{1}(t)=\mathcal{F}\left(z_{1, t}\right)+\omega_{1}(t)
$$

Then, we obtain

$$
\left\{\begin{aligned}
\dot{x}_{2}= & \varpi(t) \sin \left(\mathcal{F}\left(z_{1, t}\right)+\omega_{1}\right) \\
\dot{\omega}_{1}= & -c_{1} \psi_{1}+\psi_{2}-\dot{\mathcal{F}}(t)=-c_{1} \omega_{1}-c_{1} \mathcal{F}\left(z_{1, \tau}\right) \\
& +\psi_{2}-\mathcal{G}\left(z_{1, t}, z_{2, t}\right) \\
\dot{\psi}_{2}= & -c_{2} \psi_{2}+v_{3}
\end{aligned}\right.
$$

Let

$$
\psi_{2}(t)=\omega_{2}(t)+c_{1} \mathcal{F}\left(z_{1, t}\right)+\mathcal{G}\left(z_{1, t}, z_{2, t}\right)
$$

Then

$$
\left\{\begin{aligned}
\dot{x}_{2}= & \varpi(t) \sin \left(\mathcal{F}\left(z_{1, t}\right)+\omega_{1}\right) \\
\dot{\omega}_{1}= & -c_{1} \omega_{1}+\omega_{2} \\
\dot{\omega}_{2}= & -c_{2} \psi_{2}-c_{1} \mathcal{G}\left(z_{1, t}, z_{2, t}\right)-\ddot{\mathcal{F}}(t)+v_{3} \\
= & -c_{2} \omega_{2}(t)-c_{2} c_{1} \mathcal{F}\left(z_{1, t}\right)+v_{3}(t) \\
& -\left(c_{1}+c_{2}\right) \mathcal{G}\left(z_{1, t}, z_{2, t}\right)-\mathcal{H}\left(t, z_{1, t}, z_{2, t}, x_{2, t}\right)
\end{aligned}\right.
$$

Now, let

$$
\begin{aligned}
v_{3}(t)= & c_{2} c_{1} \mathcal{F}\left(z_{1, t}\right)+\left(c_{1}+c_{2}\right) \mathcal{G}\left(z_{1, t}, z_{2, t}\right) \\
& +\mathcal{H}\left(t, z_{1, t}, z_{2, t}, x_{2, t}\right)
\end{aligned}
$$

Then, we obtain

$$
\left\{\begin{array}{l}
\dot{x}_{2}=\varpi(t) \sin \left(\mathcal{F}\left(z_{1, t}\right)+\omega_{1}\right) \\
\dot{\omega}_{1}=-c_{1} \omega_{1}(t)+\omega_{2}(t) \\
\dot{\omega}_{2}=-c_{2} \omega_{2}(t)
\end{array}\right.
$$

Since the $\left(\omega_{1}, \omega_{2}\right)$-subsystem of $(64)$ is exponentially stable, this leads us to study the stability of:

$$
\left\{\begin{array}{c}
\dot{x}_{2}=\varpi(t) \sin \left(\mathcal{F}\left(z_{1, t}\right)\right)+\delta_{1}(t) \\
\dot{z}_{1}=q\left[-z_{1}(t)+z_{2}(t)\right] \\
\dot{z}_{2}=q\left[-z_{2}(t)-\sigma_{\dagger}\left(y_{2}(t)\right)\right]
\end{array}\right.
$$

with $\delta_{1}$ such that $\lim _{t \rightarrow+\infty}\left|\delta_{1}(t)\right|=0$.

By integrating the $z$-subsystem, we obtain

$$
z_{2}(t)=-q \int_{0}^{t} e^{q(\ell-t)} \sigma_{\dagger}\left(y_{2}(\ell)\right) d \ell+e^{-q t} z_{2}(0)
$$

and

$$
z_{1}(t)=q \int_{0}^{t} e^{q(m-t)} z_{2}(m) d m+e^{-q t} z_{1}(0)
$$

Consequently,

$$
z_{1}(t)-e^{-\tau q} z_{1}(t-\tau)=q \int_{t-\tau}^{t} e^{q(m-t)} z_{2}(m) d m
$$

From this equality and (66), we deduce that

$$
\begin{aligned}
z_{1}(t)= & e^{-\tau q} z_{1}(t-\tau) \\
= & q \int_{t-\tau}^{t} e^{q(m-t)}\left[-q \int_{0}^{m} e^{q(\ell-m)} \sigma_{\dagger}\left(y_{2}(\ell)\right) d \ell\right. \\
& \left.+e^{-q m} z_{2}(0)\right] d m \\
= & -q^{2} \int_{t-\tau}^{t} e^{q(m-t)} \int_{0}^{m} e^{q(\ell-m)} \sigma_{\dagger}\left(y_{2}(\ell)\right) d \ell d m \\
& +\delta_{2}(t)
\end{aligned}
$$

with $\delta_{2}(t)=q \tau e^{-q t} z_{2}(0)$. It follows that

$$
\begin{aligned}
z_{1}(t)= & e^{-\tau q} z_{1}(t-\tau) \\
= & -q^{2} \int_{t-\tau}^{t} e^{q(m-t)} \int_{m-\tau}^{m} e^{q(\ell-m)} \sigma_{\dagger}\left(y_{2}(\ell)\right) d \ell d m \\
& -q^{2} \int_{t-\tau}^{t} e^{q(m-t)} \int_{0}^{m-\tau} e^{q(\ell-m)} \sigma_{\dagger}\left(y_{2}(\ell)\right) d \ell d m \\
& +\delta_{2}(t) \\
= & -q^{2} \int_{t-\tau}^{t} e^{q(m-t)} \int_{m-\tau}^{m} e^{q(\ell-m)} \sigma_{\dagger}\left(y_{2}(\ell)\right) d \ell d m \\
& -q^{2} \int_{t-2 \tau}^{t-\tau} e^{q(s-t)} \int_{0}^{s} e^{q(\ell-s)} \sigma_{\dagger}\left(y_{2}(\ell)\right) d \ell d s \\
& +\delta_{2}(t) \\
= & -q^{2} \int_{t-\tau}^{t} e^{q(m-t)} \int_{m-\tau}^{m} e^{q(\ell-m)} \sigma_{\dagger}\left(y_{2}(\ell)\right) d \ell d m \\
& -q^{2} e^{-q \tau} \int_{t-\tau}^{t-\tau} e^{q(s-t+\tau)} \int_{0}^{s} e^{q(\ell-s)} \times \\
& \sigma_{\dagger}\left(y_{2}(\ell)\right) d \ell d s+\delta_{2}(t)
\end{aligned}
$$

Using (69), we obtain

$$
\begin{gathered}
z_{1}(t)-e^{-\tau q} z_{1}(t-\tau)=-q^{2} \int_{t-\tau}^{t} e^{q(m-t)} \times \\
\int_{m-\tau}^{m} e^{q(\ell-m)} \sigma_{\dagger}\left(y_{2}(\ell)\right) d \ell d m \\
+e^{-q \tau}\left[z_{1}(t-\tau)-e^{-\tau q} z_{1}(t-2 \tau)\right]+\delta_{3}(t)
\end{gathered}
$$

where $\delta_{3}$ is such that $\lim _{t \rightarrow+\infty}\left|\delta_{3}(t)\right|=0$. As an immediate consequence,

$$
\begin{aligned}
& z_{1}(t)-2 e^{-\tau q} z_{1}(t-\tau)+e^{-2 q \tau} z_{1}(t-2 \tau)=-q^{2} \times \\
& \int_{t-\tau}^{t} e^{q(m-t)} \int_{m-\tau}^{m} e^{q(\ell-m)} \sigma_{\dagger}\left(y_{2}(\ell)\right) d \ell d m+\delta_{3}(t)
\end{aligned}
$$

From (53), it follows that

$$
\begin{aligned}
\frac{\mathcal{F}\left(z_{1, t}\right)}{c_{\triangle}}= & -q^{2} \int_{t-\tau}^{t} e^{q(m-t)} \int_{m-\tau}^{m} e^{q(\ell-m)} \times \\
& \sigma_{\dagger}\left(y_{2}(\ell)\right) d \ell d m+\delta_{3}(t)
\end{aligned}
$$

Returning to (65), we obtain:

$$
\begin{aligned}
\dot{x}_{2}= & \varpi(t) \sin \left(c _ { \triangle } \left[-q^{2} \int_{t-\tau}^{t} \int_{m-\tau}^{m} e^{q(\ell-t)} \times\right.\right. \\
& \left.\left.\sigma_{\dagger}\left(y_{2}(\ell)\right) d \ell d m\right]\right)+\delta_{4}(t) \\
\triangleq & \varpi(t)(t) \sin \left(\xi\left(y_{2, t}\right)\right)+\delta_{4}(t)
\end{aligned}
$$

where $\delta_{4}$ is such that $\lim _{t \rightarrow+\infty}\left|\delta_{4}(t)\right|=0$. From (52) and (56), we easily deduce that

$$
\left|\xi\left(y_{2, t}\right)\right| \leq \frac{\pi}{4}
$$

Moreover, let us rewrite (74) as follows:

$$
\begin{aligned}
\dot{x}_{2}= & -\theta(t) c_{\triangle} q^{2} \int_{t-\tau}^{t} \int_{s-\tau}^{s} e^{q(\ell-t)} \sigma_{\dagger}\left(\eta(\ell) x_{2}(\ell)\right) d \ell d s \\
& +\delta_{4}(t) \\
= & -\theta(t) c_{\triangle} q^{2} \int_{t-\tau}^{t} \int_{s-\tau}^{s} e^{q(\ell-t)} \sigma_{\dagger}\left(\eta(\ell) x_{2}(\ell)\right) d \ell d s \\
& +\delta_{4}(t)
\end{aligned}
$$

with $\theta(t)=\varpi(t) \frac{\sin \left(\xi\left(y_{2, t}\right)\right)}{\xi\left(y_{2, t}\right)}$ when $\xi\left(y_{2, t}\right) \neq 0$ and $\theta(t)=0$ when $\xi\left(y_{2, t}\right)=0$. Then, recalling that $\varpi(t) \in\left[\frac{1}{2}, 1\right]$ and using the fact that $\sup _{m \in\left(0, \frac{\pi}{4}\right]} \frac{\sin (m)}{m} \in\left[\frac{2 \sqrt{2}}{\pi}, 1\right]$, it follows that $\theta(t) \in[\underline{\theta}, \bar{\theta}]$ with $\underline{\theta}=\frac{\sqrt{2}}{\pi}$ and $\bar{\theta}=1$. 
From (76), we deduce that

$$
\begin{aligned}
\dot{x}_{2}= & -\theta(t) c_{\triangle} q^{2} \int_{t-\tau}^{t} \int_{m-\tau}^{m} e^{q(\ell-t)} \sigma_{\dagger}\left(\eta(\ell) x_{2}(t)\right. \\
& \left.+\eta(\ell) \int_{t}^{\ell} \dot{x}_{2}(s) d s\right) d \ell d m+\delta_{4}(t)
\end{aligned}
$$

From (51), (56) and (77), it is not difficult to see that for all $\ell \in[t-2 \tau, t]$,

$$
\left|\int_{t}^{\ell} \dot{x}_{2}(s) d s\right| \leq 2 \tau \bar{\theta} \varsigma_{1} \varsigma_{2}+2 \tau \sup _{s \in[t-2 \tau, t]}\left|\delta_{4}(s)\right|
$$

Since $\lim _{t \rightarrow+\infty}\left|\delta_{4}(t)\right|=0$, we deduce that if $2 \tau \bar{\theta} \varsigma_{1}<1$, there is $t_{a} \geq 0$ such that for all $t \geq t_{a}, \sigma_{+}\left(\eta(t) x_{2}(t)\right)=$ $\varsigma_{3} \eta(t) x_{2}(t)$. To ease the following computations writing, let us note $\varsigma_{4}=\varsigma_{1} \varsigma_{3}$. Then, for all $t \geq t_{b}$,

$$
\begin{aligned}
\dot{x}_{2}= & -\theta(t) c_{\triangle} \varsigma_{4} q^{2} \int_{t-\tau}^{t} \int_{m-\tau}^{m} e^{q(\ell-t)} \eta(\ell) x_{2}(\ell) d \ell d m \\
& +\delta_{4}(t)
\end{aligned}
$$

and

$$
\begin{aligned}
\dot{x}_{2}= & -\theta(t) c_{\triangle} \varsigma_{4} q^{2} \int_{t-\tau}^{t} \int_{m-\tau}^{m} e^{q(\ell-t)}\left(\eta(\ell) x_{2}(t)\right. \\
& \left.+\eta(\ell) \int_{t}^{\ell} \dot{x}_{2}(s) d s\right) d \ell d m+\delta_{4}(t)
\end{aligned}
$$

Then, for all $t \geq t_{b}$,

$$
\begin{aligned}
\dot{x}_{2}= & -\theta(t) c_{\triangle} q^{2} \varsigma_{4} \int_{t-\tau}^{t} \int_{m-\tau}^{m} e^{q(\ell-t)} \eta(\ell) d \ell d m x_{2}(t) \\
& -\theta(t) c_{\triangle} q^{2} \varsigma_{4} \int_{t-\tau}^{t} \int_{m-\tau}^{m} e^{q(\ell-t)} \eta(\ell) \times \\
& \int_{t}^{\ell} \dot{x}_{2}(s) d s d \ell d m+\delta_{4}(t)
\end{aligned}
$$

Next, let us consider the positive definite quadratic function $V\left(x_{2}\right)=\frac{1}{2} x_{2}^{2}$. Its derivative along the trajectories of (81) satisfies

$$
\begin{aligned}
\dot{V}(t)= & -\theta(t) c_{\triangle} q^{2} \varsigma_{4} \int_{t-\tau}^{t} \int_{m-\tau}^{m} e^{q(\ell-t)} \eta(\ell) d \ell d m x_{2}(t)^{2} \\
& -x_{2}(t) \theta(t) c_{\triangle} q^{2} \varsigma_{4} \int_{t-\tau}^{t} \int_{m-\tau}^{m} e^{q(\ell-t)} \eta(\ell) \times \\
& \int_{t}^{\ell} \dot{x}_{2}(s) d s d \ell d m+x_{2}(t) \delta_{4}(t)
\end{aligned}
$$

Using the lower bound of $\eta$ and the definition of $c_{\triangle}$, we obtain

$$
\begin{aligned}
\dot{V}(t) \leq & -\theta(t) \varsigma_{4} \underline{\eta} x_{2}(t)^{2}+\theta(t) c_{\triangle} q^{2} \varsigma_{4} \bar{\eta} \times \\
& \int_{t-\tau}^{t} \int_{m-\tau}^{m} e^{q(\ell-t)} \int_{\ell}^{t}\left|x_{2}(t) \dot{x}_{2}(s)\right| d s d \ell d m \\
& +x_{2}(t) \delta_{4}(t) \\
\leq & -\theta(t) \varsigma_{4} \underline{\eta} x_{2}(t)^{2}+\theta(t) c_{\triangle} q^{2} \varsigma_{4} \bar{\eta} \times \\
& \int_{t-\tau}^{t} \int_{m-\tau}^{m} e^{q(\ell-t)} \int_{t-2 \tau}^{t}\left|x_{2}(t) \dot{x}_{2}(s)\right| d s d \ell d m \\
& +x_{2}(t) \delta_{4}(t) \\
\leq & -\theta(t) \varsigma_{4} \underline{\eta} x_{2}(t)^{2}+\theta(t) \varsigma_{4} \bar{\eta} \int_{t-2 \tau}^{t}\left|x_{2}(t) \dot{x}_{2}(s)\right| d s \\
& +x_{2}(t) \delta_{4}(t)
\end{aligned}
$$

Thus, from Young's inequality, we deduce that $\left|x_{2}(t) \dot{x}_{2}(s)\right| \leq \frac{1}{4 \tau} x_{2}(t)^{2}+\tau \dot{x}_{2}(s)^{2}$. It follows that

$$
\begin{aligned}
\dot{V}(t) \leq & -\theta(t) \varsigma_{4} \underline{\eta} x_{2}(t)^{2}+\theta(t) \varsigma_{4} \bar{\eta} \int_{t-2 \tau}^{t}\left(\frac{1}{4 \tau} x_{2}(t)^{2}\right. \\
& \left.+\tau \dot{x}_{2}(s)^{2}\right) d s+x_{2}(t) \delta_{4}(t) \\
\leq & -\frac{1}{2} \theta(t) \varsigma_{4} \bar{\eta} x_{2}(t)^{2}+\tau \bar{\theta} \varsigma_{4} \bar{\eta} \int_{t-2 \tau}^{t} \dot{x}_{2}(s)^{2} d s \\
& +x_{2}(t) \delta_{4}(t)
\end{aligned}
$$

Let

$$
W_{1}\left(t, x_{2, t}\right)=V\left(x_{2}(t)\right)+\tau \bar{\theta} \varsigma_{4} \bar{\eta} \int_{t-2 \tau}^{t} \int_{a}^{t} \dot{x}_{2}(s)^{2} d s d a
$$

An elementary calculation gives

$$
\begin{aligned}
\dot{W}_{1}(t) \leq & -\frac{1}{2} \theta(t) \varsigma_{4} \eta_{x_{2}}(t)^{2}+2 \tau^{2} \bar{\theta} \varsigma_{4} \bar{\eta} \dot{x}_{2}(t)^{2} \\
& +x_{2}(t) \delta_{4} \overline{(t)} \\
= & -\frac{1}{2} \theta(t) \varsigma_{4} \underline{\eta} x_{2}(t)^{2}+2 \tau^{2} \bar{\theta} \varsigma_{4} \bar{\eta}\left(-\theta(t) c_{\triangle} q^{2} \times\right. \\
& \left.\int_{t-\tau}^{t} \int_{s-\tau}^{s} e^{q(\ell-t)} \varsigma_{4} \eta(\ell) x_{2}(\ell) d \ell d s+\delta_{4}(t)\right)^{2} \\
& +x_{2}(t) \delta_{4}(t) \\
\leq & -\frac{1}{2} \theta(t) \varsigma_{4} \underline{\eta}_{2}(t)^{2}+2 \tau^{2} \bar{\theta} \varsigma_{4} \bar{\eta}\left(\theta(t) c_{\triangle} q^{2} \times\right. \\
& \left.\int_{t-\tau}^{t} \int_{s-\tau}^{s} e^{q(\ell-t)} \varsigma_{4} \eta(\ell) x_{2}(\ell) d \ell d s\right)^{2}+\delta_{5}(t)
\end{aligned}
$$

where $\delta_{5}$ is such that $\lim _{t \rightarrow+\infty}\left|\delta_{5}(t)\right|=0$, where the last inequality is a consequence of the fact that $x_{2}$ is bounded. Then

$$
\begin{aligned}
\dot{W}_{1}(t) \leq & -\frac{1}{2} \theta(t) \varsigma_{4} \underline{\eta} x_{2}(t)^{2}+2 \tau^{2} \bar{\theta}^{3} c_{\triangle}^{2} \varsigma_{4}^{3} \bar{\eta}^{3} q^{4} \times \\
& \left(\int_{t-\tau}^{t} \int_{s-\tau}^{s} e^{q(\ell-t)}\left|x_{2}(\ell)\right| d \ell d s\right)^{2}+\delta_{5}(t) \\
\leq & -\frac{1}{2} \underline{\theta} \varsigma_{4} \underline{\eta} x_{2}(t)^{2}+2 \tau^{2} \bar{\theta}^{3} c_{\triangle}^{2} \varsigma_{4}^{3} \bar{\eta}^{3} q^{4} \times \\
& \left(\int_{t-\tau}^{t} \int_{s-\tau}^{s}\left|x_{2}(\ell)\right| d \ell d s\right)^{2}+\delta_{5}(t) \\
\leq & -\frac{1}{2} \underline{\theta} \varsigma_{4} \underline{\eta} x_{2}(t)^{2}+2 \tau^{4} \bar{\theta}^{3} c_{\triangle}^{2} \varsigma_{4}^{3} \bar{\eta}^{3} q^{4} \times \\
& \left(\int_{t-2 \tau}^{t}\left|x_{2}(\ell)\right| d \ell\right)^{2}+\delta_{5}(t) \\
\leq & -\frac{1}{2} \underline{\theta} \varsigma_{4} \underline{\eta}_{2} x_{2}(t)^{2}+4 \tau^{5} \bar{\theta}^{3} c_{\triangle}^{2} \varsigma_{4}^{3} \bar{\eta}^{3} q^{4} \times \\
& \int_{t-2 \tau}^{t} x_{2}(\ell)^{2} d \ell+\delta_{5}(t)
\end{aligned}
$$

where the last inequality is a consequence of CauchySchwarz's inequality. Let

$$
\begin{aligned}
W_{2}\left(t, x_{2, t}\right)= & W_{1}\left(t, x_{2, t}\right) \\
& +\frac{4}{\underline{\theta}} \tau^{5} \bar{\theta}^{3} c_{\triangle}^{2} \varsigma_{4}^{3} \bar{\eta}^{3} q^{4} \int_{t-2 \tau}^{t} \int_{s}^{t} x_{2}(\ell)^{2} d \ell d s
\end{aligned}
$$

Then

$$
\dot{W}_{2}(t) \leq \frac{1}{2} \varsigma_{4}\left[-\underline{\theta} \underline{\eta}+16 \bar{\theta}^{3} c_{\triangle}^{2} \varsigma_{4}^{2} \bar{\eta}^{3} q^{4} \tau^{6}\right] x_{2}(t)^{2}+\delta_{5}(t)
$$

This allows us to conclude that if $\tau$ is such that

$$
\tau^{6}<\frac{\underline{\underline{\eta}} \underline{\left(1-e^{-q \tau}\right)^{4}}}{16 \bar{\theta}^{3} \varsigma_{4}^{2} \bar{\eta}^{3} q^{4}}
$$

then $x_{2}(t)$ converges to zero. Then it follows that $z_{1}(t)$ and $z_{2}(t)$ converge to zero. 